\title{
The efficacy of tamoxifen during frozen embryo transfer cycles in women with thin endometrium
}

\author{
Jingjuan $\mathrm{Ji}^{1}$, lingli Huang ${ }^{1}$, and lihua Luo ${ }^{1}$ \\ ${ }^{1}$ Affiliation not available
}

April 28, 2020

\begin{abstract}
Objective: To investigate whether live birth rate (LBR) following frozen-thawed embryo transfer in tamoxifen -stimulated cycles (T-FET) differs from hormone replacement treatment FET (HRT- FET) in women with thin endometrium. Design: Retrospective cohort study. Setting: Tertiary-care academic medical center. Participant(s): A total of 671 patients with thin endometrium who fulfilled the inclusion criteria were involved in the period from January 2016 till February 2019. Methods In the group of T-FET, $20 \mathrm{mg}$ TAM per day was giving from day 5 of the menstrual cycle for 5 days. Day-3 ET was performed four days after ovulation while blastocyst transfer was performed six days after ovulation. In the group of HRT- FET, estradiol valᄀerate was taken $6 \mathrm{mg} / \mathrm{d}$ from menstrual cycle day 2-3. 12 to 14 days later progesterone 40 - $60 \mathrm{mg} / \mathrm{d}$ was given. Embryo transfer was performed 3 or 5 days later for day-3 embryos or blastocysts respectively. Main Outcome Measure(s): LBR per embryo transfer was the primary outcome. The secondary end points included ongoing and clinical pregnancy rate, cancellation rate, endometrial thickness and pregnancy loss rate. Multivariable logistic regression analysis was performed to adjust for potential confounders. Result(s): LBR was significantly higher in T-FET group than HRT-FET group. Moreover, the clinical and ongoing pregnancy rate also higher in the T-FET group than in the HRT-FET group. Conclusion(s): In patients with thin endometrium undergoing FET, tamoxifen use for endometrial preparation was associated with higher LBR compared with HRT cycles. Funding: No external funding was used.
\end{abstract}

\section{Introduction}

Being one of the key parameters of endometrial receptivity, endometrial thickness plays an important role in successful embryo implantation (1).Although there has been no commonly accepted thickness below which successful implantation would be preclude, it has been recognized by many investigators that endometrial thickness [?]7mm on the day of luteinizing hormone(LH) surge or human chorionic gonadotrophin(HCG) administration is preferred for embryo transfer $(2-5)$. Thin endometrium $(i 7 \mathrm{~mm})$ affects about $2.4 \%$ patients in in vitro fertilization(IVF) cycles (6).It often results in cancelation of embryo transfer and cryopreservation of all the embryos.

Various strategies have been attempted to improve endometrial growth in these patients, including estrogen supplementation, vasoactive measures such as vaginal sildenafil, low-dose aspirin, pentoxifylline, or tocopherol, intrauterine infusion of granulocyte colony-stimulating factor, and regenerative medicine(7-11). Among them, exogenous estrogen treatment is the most commonly used method(12). Many factors may influence the effectiveness of estrogen treatment and the subsequent reproductive outcomes(12-14).Although high dose estrogen treatment sustaining a long time may be effective for some of these patients(12), supraphysiologic estrogen levels resulted from this treatment may have a negative impact on reproductive outcomes(15-19). Moreover, a higher risk of side-effects, such as breakthrough bleeding (20), some types of tumor such as endometrial cancer (21), deep venous thrombosis (22) may also be associated with this treatment. Furthermore, after pregnancy, exogenous estrogen and progesterone should be continued until 8-10 weeks of gestation to substitute for the absent corpus luteum. In addition, some patients were insensitive to estrogen treatment. 
Alternative methods should be considered for endometrium preparation in these women during their frozen embryo transfer (FET) cycles.

Tamoxifen (TAM) is a selective estrogen receptor modulator (SERM) with chemical properties similar to clomiphene citrate (CC).It has been used in clinic as a highly effective agent for the treatment of breast cancer(23)and anovulation(24-26). In contrast to CC, whose anti-estrogenic effects at the level of the endometrium sometimes result in thin endometrium(27), ovulation induction with tamoxifen improves endometrial thickness in women with a history of thin endometrium (28). However, little information is available regarding its use during FET cycles in that kind of patients. To the best of our knowledge, there has been three published study investigating the use of TAM for endometrium preparation (EP) in thin endometrium patients. The first one is only a case report (29). The obvious weakness of the other two studies is that there were no control groups $(30,31)$. Furthermore, they are limited by their small sample size. The aim of this study is to evaluate the efficacy of TAM in women with thin endometrium during their FET cycles through comparing it with the most commonly used protocol- hormone replacement treatment (HRT) protocol. We propose that the result of our study may contribute to the treatment of infertility patients with thin endometrium.

\section{Methods}

\section{Study design}

This retrospective study was performed at the Reproductive Medical Center of Anhui Provincial Hospital, involving women with thin endometrium who undergoing their FET cycles during the period from January 2016 till February 2019.

In our center, thin endometrium is diagnosed when the maximal endometrial thickness(EMT) is i7 mm, dominant follicle are $18 \mathrm{~mm}$ in diameter in nature cycle(NC), or after 12 to 16 days of estradiol (E2) replacement(4-6 mg, Progynova; Bayer Schering Pharma, Roubaix, France). Infertile women who met these criteria in their 1-2 previously FET cycles were included in this study. All these women failed pregnancy despite the treatment with oral and/or vaginal estrogen, sildenafil or aspirin. Exclusion criteria were: uterine or endometrial abnormalities other than thin endometrium; patients with repeated implantation failures; 20 or $>40$ years of age at oocyte retrieval; patients with multiple types of FET protocol following the same fresh IVF/ICSI cycle. As there isn't any consensus as to the ideal method of endometrium preparation in patients with thin endometrium, endometrium was prepared with TAM or HRT at the discretion of physicians and/or patients' preference. The study was approved by the institutional ethics committee of Anhui Provincial Hospital.

\section{The FET cycle}

In the group of TAM, $20 \mathrm{mg}$ per day was giving from day 5 of the menstrual cycle for 5 days. Vaginal ultrasound examinations were performed on day 10 of the cycle to monitor the number and size of developing follicles and endometrial thickness. Ovulation was induced with 10,000 I.U. hCG (human chorionic gonadotropin, Lizhu Pharmaceutical Trading Co.) when the leading follicle reached 18-22 $\mathrm{mm}$ and the endometrium thickness reached $7 \mathrm{~mm}$. Day-3 ET was performed four days later while blastocyst transfer was performed six days later. Exogenous progesterone $(20 \mathrm{mg} / \mathrm{d}$; Duphaston; Abbott Biologicals B.V.,

Netherlands) was given vaginally starting 2 days after hCG administration.If no dominant follicle developed, a daily dosage of $75 \mathrm{IU}$ hMG (human menopausal gonadotropin, Lizhu Pharmaceutical Trading Co.) was given from day 10 up to hCG injection, with incremental doses of $37.5 \mathrm{IU}$ if needed. If there was no dominant follicle after 15 days of ovarian stimulation or if the follicle reached a diameter of maturity and endometrial thickness did not attain $7 \mathrm{~mm}$, the cycle would be cancelled. If the endometrial thickness did not attain 7 $\mathrm{mm}$ when follicle reached a diameter of maturity, the cycle would be cancelled either.

In the group of hormone replacement treatment cycle, oral estradiol valerate (progynova, Schering, German) was taken $6 \mathrm{mg} / \mathrm{d}$ from menstrual cycle day 2-3. An ultrasound assessment was done 12 to 14 days later to assess endometrium thickness. Progesterone $40 \mathrm{mg} / \mathrm{d}$, which would be changed to $60 \mathrm{mg} / \mathrm{d} 2$ days later, was 
given to transform the endometrium, provided the endometrial thickness exceeded $7 \mathrm{~mm}$. Embryo transfer was performed 3 days after progesterone administration for day- 3 embryos or 5 days later for blastocysts. If the endometrium thickness is not adequate, endometrial preparation continued with step-up dose of E2 or adding vaginal estradiol (Femoston, Solvay pharmaceuticals B.V.) $1-2 \mathrm{mg} / \mathrm{d}$ till the endometrium thickness reaching $7 \mathrm{~mm}$. Cycles were canceled in patients whose endometrial thickness remained $<7 \mathrm{~mm}$ after 21 days of continuous estradiol administration.

In both TAM-FET and HRT-FET groups, luteal support was continued to 10 weeks of gestation if a pregnancy achieved.

\section{Embryo vitrification and thawing}

For Day 3 embryos, our laboratory procedure of vitrification and warming was the same as the method used for human oocytes as reported by Tong et al. previously (32). For blastocysts, a glass micro-needle was used to collapse the blastocyst before vitrification. The following steps were the same as for the Day 3 embryos.

Embryo quality was graded as 1(good), 2(reasonable), or 3(moderate) according to the number of cells, degree of fragmentation and renewed development of the embryo. This standard was based on the ESHRE Istanbul consensus on embryo assessment (33).

\section{Definition of pregnancy}

A serum $\beta$-hCG assay 11-14 days after ET. Clinical pregnancy was defined as the presence of a gestational sac on transvaginal ultrasound.

Clinical miscarriage was defined if the pregnancy terminated before 12 weeks of gestational age. Implantation rate was defined as the number of intrauterine sacs divided by the number of embryos transferred.

Ongoing pregnancy was defined as gestations that reached 20 weeks or more. Live birth was defined as give birth to an infant [?]24 weeks' gestation.

\section{Statistical analysis}

All analyses have been performed using IBM Spss statistics 21.For continuous variables, Student's t-test and Mann-Whitney test were used for data with homogeneous variance and heterogeneous variance respectively. The $\mathrm{x}^{2}$ test was used for categorical variables. Logistic regression analyses were conducted to identify independent correlates between each possible confounding factor, especially protocols for endometrium preparation and pregnancy outcome after adjusting for other confounders that were identified in our univariate analysis. A p-value $<0.05$ was considered statistically significant.

\section{Results}

\section{Study Population}

We analyzed 671 women who fulfilled the inclusion criteria from our unit, with no loss to follow-up. Of all the patients, 250 women underwent TAM treatment and 421 women underwent hormone replacement therapy (HRT). 42 cycles in TAM group and 76 cycles in HRT group were canceled. There were 28 cycles in TAM group and 58 cycles in HRT group been canceled due to insufficient endometrial thickness.

In the TAM group, insufficient follicle development was seen in 8 women and 4 women experienced a premature progesterone rise $(¿ 1.5 \mathrm{ng} / \mathrm{mL})$. No serious adverse effects were observed. The overall cancellation rate was similar between the two study groups $(\mathrm{p}=0.753$, Table 1$)$.

\section{Baseline Characteristics}

A summary of the patients' baseline characteristics is presented in Table 1. There were no significant differences in duration of infertility, BMI, proportion of primary infertility, the major indication for IVF, proportion of $\mathrm{GnRH}$ angonist long protocol between the two treatment groups. Statistically significant 
differences between the two groups were noted in terms of age, duration of ovarian stimulation and oocytes retrieved.

\section{Cycle Characteristics and Reproductive Outcomes of FET}

The Cycle Characteristics and reproductive Outcomes of FET are presented in Table 2. Endometrial thickness and its increase are significantly greater in TAM group (8.98 $\pm 2.02 \mathrm{vs} 8.15 \pm 1.11,2.82 \pm 2.21 \mathrm{vs} 2.2 \pm 1.59)$. Nonetheless, the duration of endometrium preparation was shorter in TAM group than HRT group. The distribution of best embryos transfer was comparable between the two groups $(\mathrm{p}=0.481)$. The clinical pregnancy rate, implantation rate, ongoing pregnancy rate and live birth rate were significantly higher in TAM group than HRT group. The miscarriage rate was similar between the two groups $(11.8 \%$ vs.14.0\%; p=0.701).

After adjusting the results for the above-mentioned potential confounders (Table 3), the CPRs, OPRs and LBRs remained consistently higher [adjusted odds ratio (aOR), 95\% CI] in TAM group compared with HRT group[CPR(aOR1.820, 95\% CI1.219-2.72), OPRs(aOR1.640, 95\% CI1.090-2.467) LBRs(aOR1.570, 95\% CI1.018-2.420)].

\section{Discussion}

Although various strategies have been attempted to improve endometrial growth in women with thin endometrium, the optimal means of EP in these women is yet to be determined. The aim of our study was to evaluate the effect of TAM in patients with a thin endometrium in FET cycles. By univariate and multivariate analysis, our results found a superior pregnancy outcome following FET in a TAM ovarian stimulation cycle compared to HRT cycle in women with thin endometrium.

In our study we observed that the durations of endometrium preparation were 6 day shorter in the TAM FET group (10 days) than in the HRT FET group (16 days). This phenomenon is interesting but hard to explain. We hypothesize that ovarian stimulation with TAM may stimulate a faster development of the dominant follicle(s). More prospective studies are needed to explore its clinical relevance.

The result of this study showed that endometrial lining of the current HRT cycle differ from prior cycle $(8.15 \pm 1.11 \mathrm{~mm}$ vs $¡ 7 \mathrm{~mm})$. Different from previous cycle, prolonged duration, increased dosage and transvaginal estrogen treatment were used in the present study. The study of Liu showed that effectiveness of estrogen treatment was significantly associated with treatment duration (12). Our study was in line with this study.

Our results showed that endometrium thickness was much more improved in TAM group than HRT group. TAM has been reported to improve endometrium thickness while ovulation induction. In the study of Kasey Reynolds, endometrial thickness was improved when the OI (ovulation induction) drug been switched from CC to TAM (34). Wang et al compared TAM and CC in ovarian stimulation cycle in combination with HMG (human menopausal gonadotrophin) and found that TAM treated patients had a significantly increased endometrial thickness (28).

The literature on the treatment of thin endometrium with TAM in FET cycle is rather sparse. Chen X et al used TAM in 3 women who showed a repeated unresponsive thin endometrium and resulted in endometrium expansion to at least $7.7 \mathrm{~mm}$ and conception(29). Soon after, another study by the same group showed TAM increased the endometrial thickness from 6.5 to $8.8 \mathrm{~mm}$ in women with thin endometrium undergoing FET(30). Ke $\mathrm{H}$ et al explore the effect of TAM in patients with a thin endometrium in FET cycle. Their study included 226 women who had an EMT of less than $7.5 \mathrm{~mm}$ in their previous cycles. After the use of TAM, EMTs were significantly improved. When stratified by different previous endometrium preparation protocols, EMT increased from $6.11+-0.98 \mathrm{~mm}$ to $7.87+-1.48$ in NC group, from $6.24+-1.01$ to $8.22+-1.67$ in HRT group and from $6.34+-1.03$ to $8.05+-1.58 \mathrm{~mm}$ in OI group(31). In line with the previous data, our result showed that the EMT in TAM group was $8.98+-2.02 \mathrm{~mm}$ which was significantly thicker than the EMT in HRT groups $(8.15+-1.11 \mathrm{~mm})$.

Some authors found that TAM exposure promotes endometrial cell proliferation through estrogen and non- 
estrogen pathway. In estrogen signaling pathway, TAM stimulate the GPER (GPR30 Estrogen Receptor), which in turn, activates the SF1 (Steroidogenic Factor 1), which is a transcription factor that induce aromatase expression in endometrial cells (35). In non-estrogen signaling pathway, a study found that TAM up-regulates the expression of Ki67 and IGF-1,markers of proliferation(36).

Although the first studies by Chen X et al only include 3 recurrent thin endometrium patients who successfully conceived with one miscarriage after tamoxifen treatment(29), their following studies consisting of 61 thin endometrium women showed that the clinical pregnancy rate, early miscarriage rate and implantation rate were 44.3(2/27) - 24.2patients of recurrent thin endometrium, tamoxifen treatment may be a successful alternative approach(30). The study of $\mathrm{Ke} \mathrm{H}$ et al showed that when stratified by different etiologies of thin endometrium, patients with PCOS obtained the highest rate of clinical pregnancy (60\%) and live birth (55.56\%) per transfer. The clinical pregnancy rate and live birth rate in patients with history of intrauterine adhesion were $6 / 18(33.33 \%)$ and $(5 / 18,27.78 \%)$ respectively, while for patients with history of uterine curettage, the clinical pregnancy rate and live birth rate were $(39 / 101,38.61 \%)$ and $(32 / 101,31.68 \%)$ respectively(31). However, there were no control groups in the studies of Chen $\mathrm{X}$ et al and $\mathrm{Ke} \mathrm{H}$ et al. Furthermore, they are limited by their small sample size. Different from the previous study, our study compared the effect of TAM with the mostly commonly used protocol- HRT protocol rather than described the reproductive outcome only so that this effect can be analyzed precisely. Overcoming methodological shortcomings in above-mentioned studies and adjusting for a number of important confounders, our study demonstrated that not only the clinical pregnancy rate and the implantation rate but also the ongoing pregnancy rate and live birth rate of TAM group were significantly higher than HRT group.

Despite the improvement of endometrium thickness, another possible mechanism for improved reproductive outcomes with the use of TAM during FET is that TAM might potentially improve corpus luteum function. $\mathrm{Wu}$ reported lower spontaneous abortion rates in patients with luteal-phase dysfunction treated with TAM as compared with $\mathrm{CC}(37)$.

Cost-effectiveness is a major concern for patients during their infertility treatment. TAM is much cheaper than estrogen agents. In China, TAM costs $\$ 42$ for the whole cycle whereas estradiol valerate costs more than $\$ 11$ per day supply and cannot be withdrawn until 8-10 weeks gestation. Future studies analyzing cost efficiency of these two regimens are needed.

Long-term safety of offspring after IVF treatment is of paramount importance. The short half-life of TAM (86h) and administration of this agent in the early follicular phase from cycle day 5 to day 10 leave a sufficient period of time for complete washout before embryo implantation. Furthermore, previous research suggested that TAM administration during the first trimester did not increase the risk of major congenital anomalies (38).

We recognize there are some important limitations of our retrospective study. First, the retrospective nature of our analysis may subject to selection bias regarding the type of FET protocol (TAM vs.HRT). In this regard, we exclude multiple types of FETs following the same fresh IVF/ICSI cycle. Secondly, endometrial receptivity assay was not performed on both groups of patients. Further studies on this subject are needed to investigate the details of the effect of TAM on the endometrium.

Our study also has some strength. First, our study is the largest in this area by far with $100 \%$ follow-up and provides new insight into current practice patterns. Secondly, the laboratory conditions and IVF procedures did not been changed throughout the study period.

\section{Conclusion}

The results of this study showed that beyond endometrial expansion, TAM protocol beneficially affect pregnancy outcome in women with thin endometrium undergoing FET.

\section{Disclosure of interests}

None declared. 


\section{Contribution to authorship}

J.J. was involved in substantial contributions to conception and design, acquisition, analysis and interpretation of data, drafting and revising the article and final approval of the version to be published; L.H. was involved in contributions to analysis and interpretation of data and final approval of the version to be published; L.L. was involved in contributions to acquisition of data and final approval of the version to be published.

\section{Funding}

No external funding was used.

\section{Details of ethics approval}

Human Ethics Committee approval for the research of TAM using in women with thin endometrium came from the Institutional Review Board (IRB) of the The First Affiliated Hospital of USTC (Anhui Provincial Hospital)on 21 January 2020(no. 2020-P-003).

\section{Acknowledgements}

We thank the entire staff of the Department of Reproductive Medicine Center, the First Affiliated Hospital of USTC for their support in this study.

\section{References}

1. Kovacs P, Matyas S, Boda K, Kaali SG. The effect of endometrial thickness on IVF/ICSI outcome. Hum Reprod. 2003;18:2337-41.

2. El-Toukhy T, Coomarasamy A, Khairy M, Sunkara K, Seed P, Khalaf Y et al. The relationship between endometrial thickness and outcome of medicated frozen embryo replacement cycles. Fertil Steril. 2008;89:832-9.

3. Kumbak B, Erden HF, Tosun S, Akbas H, Ulug U, Bahçeci M. Outcome of assisted reproduction treatment in patients with endometrial thickness less than $7 \mathrm{~mm}$. Reprod Biomed Online. 2009;18:7984.

4. Zhang X, Chen CH, Confino E, Barnes R, Milad M, Kazer RR. Increased endometrial thickness is associated with improved treatment outcome for selected patients undergoing in vitro fertilizationembryo transfer. Fertil Steril. 2005;83:336-40

5. Richter KS, Bugge KR, Bromer JG, Levy MJ. Relationship between endometrial thickness and embryo implantation, based on 1,294 cycles of in vitro fertilization with transfer of two blastocyst-stage embryos. Fertil Steril. 2007;87:53-9.

6. Kasius A, Smit JG, Torrance HL, Eijkemans MJ, Mol BW, Opmeer BC et al. Endometrial thickness and pregnancy rates after IVF: A systematic review and meta-analysis. Hum Reprod Update. 2014;20:53041.

7. Lebovitz O, Orvieto R. Treating patients with "thin" endometrium - An ongoing challenge. Gynecol Endocrinol 2014;30:409-14.

8. Zhao J, Zhang Q, Wang Y, Li Y. Uterine infusion with bone marrow mesenchymal stem cells improves endometrium thickness in a rat model of thin endometrium. Reprod Sci 2015;22:181-8.

9. Rahmati M, Petitbarat M, Dubanchet S, Bensussan A, Chaouat G, Ledee N. Granulocyte-Colony Stimulating Factor related pathways tested on an endometrial ex-vivo model. PLoS One 2014;9:e102286.

10. Xu B, Zhang Q, Hao J, Xu D, Li Y. Two protocols to treat thin endometrium with granulocyte colony-stimulating factor during frozen embryo transfer cycles. Reprod Biomed Online 2015;30:349-58.

11. Chang Y, Li J, Chen Y, Wei L, Yang X, Shi Y, et al. Autologous platelet-rich plasma promotes endometrial growth and improves pregnancy outcome during in vitro fertilization. Int J Clin Exp Med 2015;8:1286-90.

12. Liu SM, Zhou YZ, Wang HB, Sun ZY, Zhen JR, Shen K, et al. Factors Associated with Effectiveness of Treatment and Reproductive Outcomes in Patients with Thin Endometrium Undergoing Estrogen Treatment. Chin Med J (Engl) 2015;128:3173-7. 
13. Liao X, Li Z, Dong X, Zhang H. Comparison between oral and vaginal estrogen usage in inadequate endometrial patients for frozen-thawed blastocysts transfer. Int J Clin Exp Pathol 2014;7:6992-7.

14. Chen MJ, Yang JH, Peng FH, Chen Su, Ho HN, Yang YS. Extended estrogen administration for women with thin endometrium in frozen-thawed in-vitro fertilization. J Assist Reprod Genet 2006;23:337-42.

15. Ma W, Song H, Das SK, Paria BC, Dey SK. Estrogen is a critical determinant that specifies the duration of the window of uterine receptivity for implantation. Proc Natl Acad Sci U S A 2003;100:2963-8.

16. Simon C, Dominguez F, Valbuena D, Pellicer A. The role of estrogen in uterine receptivity and blastocyst implantation. Trends Endocrinol Metab 2003;14:197-9.

17. Chang EM, Han JE, Kim YS, Lyu SW, Lee WS, Yoon TK. Use of the natural cycle and vitrification thawed blastocyst transfer results in better in-vitro fertilization outcome: cycle regimens of vitrification thawed blastocyst transfer. J Assist Reprod Genet 2011;28:369-74.

18. Morozov V, Ruman J, Kenigsberg D, Moodie G, Brenner S. Natural cycle cryo-thaw transfer may improve pregnancy outcome.J Assist Reprod Genet. 2007;24:119-23.

19. Pereira N, Elias RT, Christos PJ, Petrini AC, Hancock K, Lekovich JP, et al. Supraphysiologic estradiol is an independent predictor of low birth weight in full-term singletons born after fresh embryo transfer. Hum Reprod. 2017;32:1410-7.

20. Borini A, Dal Prato L, Bianchi L, Violini F, Cattoli M, Flamigni C. Effect of duration of estradiol replacement on the outcome of oocyte donation. J Assist Reprod Genet 2001;18:185-90.

21. Sjogren LL, Morch LS, Lokkegaard E. Hormone replacement therapy and the risk of endometrial cancer: A systematic review. Maturitas. 2016;91:25-35.

22. de Bastos M, Stegeman BH, Rosendaal FR, Van Hylckama Vlieg A, Helmerhorst FM, Stijnen T, et al. Combined oral contraceptives: venous thrombosis. Cochrane Database Syst Rev. 2014 Mar $3 ;(3)$ :CD010813.

23. W.C. Park, V.C. Jordan. Selective estrogen receptor modulators (SERMS) and their roles in breast cancer prevention, Trends Mol. Med. 2002; 8:82-8.

24. Jie L, Li D, Yang C, Haiying Z. Tamoxifen versus clomiphene citrate for ovulation induction in infertile women. Eur J Obstet Gynecol Reprod Biol. 2018;228:57-64.

25. Badawy A, Gibreal A. Clomiphene citrate versus tamoxifen for ovulation induction in women with PCOS: a prospective randomized trial. Eur J Obstet Gynecol Reprod Biol. 2011;159:151-4.

26. Boostanfar R, Jain JK, Mishell DR Jr, Paulson RJ. A prospective randomized trial comparing clomiphene citrate with tamoxifen citrate for ovulation induction. Fertil Steril. 2001 ;75:1024-6.

27. Sovino H, Sir-Petermann T, Devoto L. Clomiphene citrate and ovulation induction. Reprod Biomed Online 2002;4:303-10.

28. Wang H, Wang C, Horng S, et al. Ovulation induction with tamoxifen and alternate-day gonadotrophin in patients with thin endometrium. Reprod Biomed Online,2008;17:20-26

29. Chen X, Chen s. Successful pregnancy in recurrent thin endometrium with new uses for an old drug. J IVF Reprod Med Genet.2013;1(110):Doi:10.4172/jfiv.1000110

30. Tian X,Chen X,Xu L,et al. Effect of tamoxifen on clinical outcome of patients with thin endometrium undergoing frozen-thaw embryo transfer. Chin J Pract Gynecol Obstetr.2015;31:736-740

31. Ke H, Jiang J, Xia M, Tang R, Qin Y, Chen ZJ. The Effect of Tamoxifen on Thin Endometrium in Patients Undergoing Frozen-Thawed Embryo Transfer. Reprod Sci. 2018;25:861-866.

32. Tong XH, Wu LM, Jin RT, Luo LH, Luan HB, Liu YS. Fertilization rates are improved after IVF if the corona radiata is left intact in vitrified-warmed human oocytes. Hum Reprod 2012;27:3208-14.

33. Alpha Scientists in Reproductive Medicine and ESHRE Special Interest Group of Embryology. The Istanbul consensus workshop on embryo assessment: proceedings of an expert meeting. Hum Reprod 2011;26:1270-83.

34. Kasey Reynolds, Jane Khoury, James Sosnowski, Jennifer Thie, Glen Hofmann. Comparison of the effect of tamoxifen on endometrial thickness in women with thin endometrium $(<7 \mathrm{~mm})$ undergoing ovulation induction with clomiphene citrate. Fertil Steril 2010;93:2091-3.

35. Lin BC, Suzawa M, Blind RD, Tobias SC, Bulun SE, Scanlan TS, et al. Stimulating the GPR30 estrogen receptor with a novel tamoxifen analogue activates SF-1 and promotes endometrial cell proliferation. 
Cancer Res. 2009;69:5415-23.

36. Roy RN, Gerulath AH, Cecutti A, Bhavnani BR. Effect of tamoxifen treatment on the endometrial expression of human insulin-like growth factors and their receptor mRNAs. Mol Cell Endocrinol. 2000;165:173-8.

37. Wu CH. Less miscarriage in pregnancy following Tamoxifen treatment of infertile patients with luteal phase dysfunction as compared to clomiphene treatment. Early Pregnancy.1997; 3:301-305.

38. Berger JC, Clericuzio CL. Pierre Robin sequence associated with first trimester fetal tamoxifen exposure. Am J Med Genet A. 2008;146A:2141-4.

\section{Hosted file}

table. docx available at https://authorea.com/users/309441/articles/440377-the-efficacy-of-tamoxifenduring-frozen-embryo-transfer-cycles-in-women-with-thin-endometrium 